\title{
Study of Morphology of Graphene using Atomic Force Microscopy and Raman Spectroscopy
}

\author{
S. Drewniak ${ }^{* 1}$, T. Pustelny ${ }^{1}$, I. Pasternak ${ }^{2}$ A. Krajewska ${ }^{2,3}$, G. Konieczny ${ }^{1}$ \\ ${ }^{I}$ Department of Optoelectronics, Silesian University of Technology, 2A Akademicka St., 44-100 Gliwice, Poland \\ ${ }^{2}$ Institute of Electronic Materials Technology, 133 Wólczyńska St, 01-919 Poland \\ ${ }^{3}$ Institute of Optoelectronics, Military University of Technology, 2 Gen. S. Kaliskiego St, 00-908 Warsaw, Poland
}

Received November 23, 2014; accepted December 17, 2014; published December 31, 2014

\begin{abstract}
This paper presents an analysis of a graphene layer imposed on a resistance sensing structure. All the studies are directed to determine the quantity and quality of the graphene layer. We present the results of the experiments using atomic force microscopy, electron microscopy and Raman spectroscopy. The Raman spectra were subjected to detailed analyses, especially the position and half width of the $2 \mathrm{D}$ peak.
\end{abstract}

Despite the fact that graphene was already described in the early years of XX century [1], it has only recently gained popularity. Since A. Geim and K. Novoselov informed the scientific community of having obtained stable monolayers of carbon, graphene has been of intensive interest [2-3]. Many research centers all over the world are conducting research to obtain industrially available graphene of high quality. Among its unquestionable advantages are: considerable mobility of the carriers [4], considerable electrical conductivity and good optical transparence [5]. Moreover, this material is characterized by extreme mechanical strength (to $130 \mathrm{GPa}$ [6]) and large elasticity of Young's module (about 1TPa [7]).

In recent years, there has been significant development of new methods of preparation and modification of nanomaterials [8]. The main applied methods of grapheme production are: 1) deposition of carbon layers from the gaseous phase (CVD) [9-12], 2) extraction of carbon layers on carbon silicate [12-14], 3) reduction of graphite oxide [12] and 4) exfoliation of graphite [12]. The first two methods belong to the co-called Bottom-Up group, second two - to the Top-Down group [15]. The present paper deals with an analysis of graphene obtained by means of the first method, which permits to get this material on various substrates.

One of the methods permitting a detailed characterization of graphene is Raman's spectroscopy. Figure 1 presents a typical spectrum of graphene (obtained by using the platform N-TEGRA Spectra, NTMDT). Raman's spectrum of graphene consists of three characteristic peaks: D situated within the range of the

http://www.photonics.pl/PLP wave number $\sim 1350 \mathrm{~cm}^{-1}, \mathrm{G}-$ situated in the range $\sim 1580 \mathrm{~cm}^{-1}$ and 2D situated in the range $\sim 2700 \mathrm{~cm}^{-1}$.

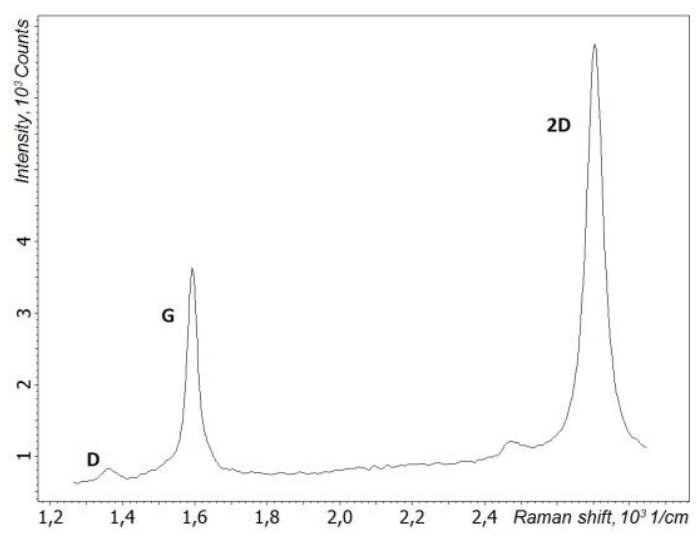

Fig. 1. Raman's spectrum of graphene.

Each of these peaks occurs as a result of other phenomena taking place inside the crystallographic structure of graphene. Peak D appears due to the lack of well-ordered atoms or the presence of defects in the atomic lattice [1718 ], peak $G$ is the consequence of vibrations of atoms along the plane of the layer [18], and 2D is caused by vibrations of atoms perpendicularly to the plane of graphene [17-18]. The latter one is also often used to identify the amount of carbon layers in the structure [16].

The aim of investigations presented in this paper was describing graphene with regard to its quality and the way of its arragement on the substrates. The applied graphene was produced at ITME (Instutite of Electronic Materials Technology) in Warsaw. The investigations were carried out at Optoelectronic Department engaged in using resistive structures in detecting selected (e.g. hydrogen) gases in synthetic air or in nitrogen. The investigations accomplished so far indicate that adequately modified graphene may prove to be sensitive to gases in sensors of this type. A simplified diagram of a basic resistant structure has been presented in Fig. 2a. On a silicon substrate with an oxidized $\mathrm{SiO}_{2}$ surface, gold electrodes 
were obtained using the PVD method. In order to improve the adhesion of gold to the substrate, a layer of chromium with a thickness of several nanometers was used. A part of this structure, imaged by means of atomic force microscopy (AFM) can be seen in Fig. 2 b.
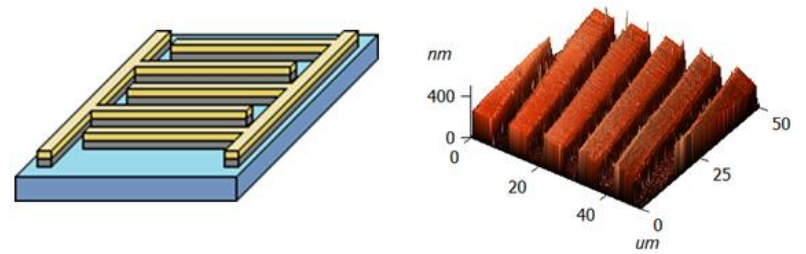

Fig. 2. a) Simplified digram of a resistant structure; b) topography of the actual structure, obtained by means of atomic force microscopy (AFM).

The technique of chemical vapour deposition CVD on $25 \mu \mathrm{m}$ copper foil and then transfering it on a basic structure was described in paper [9].

Graphene has been characterized by atomic force microscopy and Raman's spectroscopy. For this purpose the N-TEGRA Spectra platform have been used (applying VIT-P cantilevers and laser wavelength equal 532nm). Measurements have been performed in various points of the transducer in order to obtain reliable results.

First, the topography image (Fig. 3a) and phase shift image (Fig. 3b), illustrating changes in the physical properties, was made. The images of the topography show fragments of two electrodes and the surface between them. Between electrodes, graphene is suspended (wholly or partially). Fig. 4 shows the cross-section of the area marked in Figs. 3a and 3b.
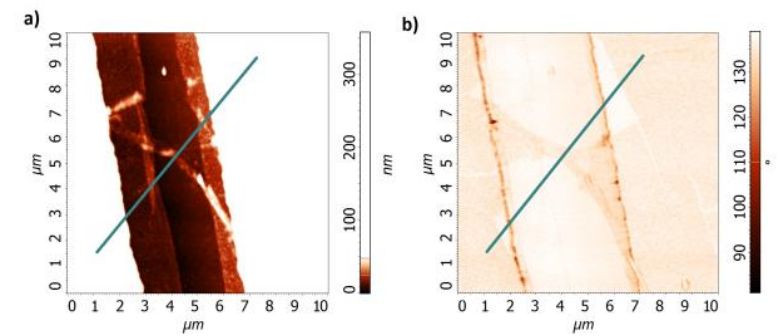

Fig. 3. a) The topography of the surface covered by graphene layer, b) the phase shift image of the same area.

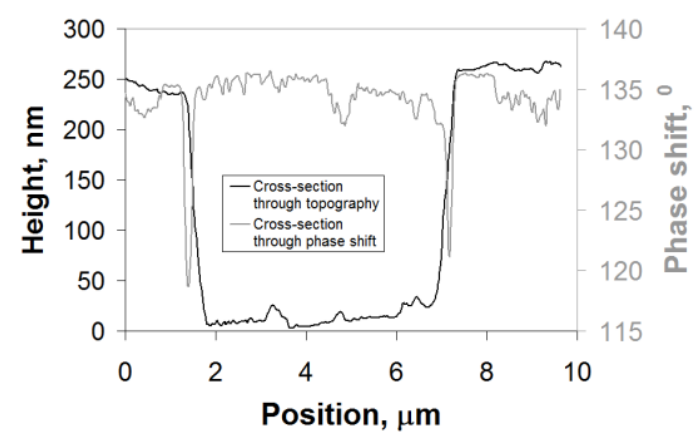

Fig. 4. The cross-section by the line selected in Figs. 3a-b.

In some areas, the layer of graphene is rolling. It has also been confirmed by electron microscopy (in some places on electrodes). A fragment of the analyzed structure is shown in Fig. 5.

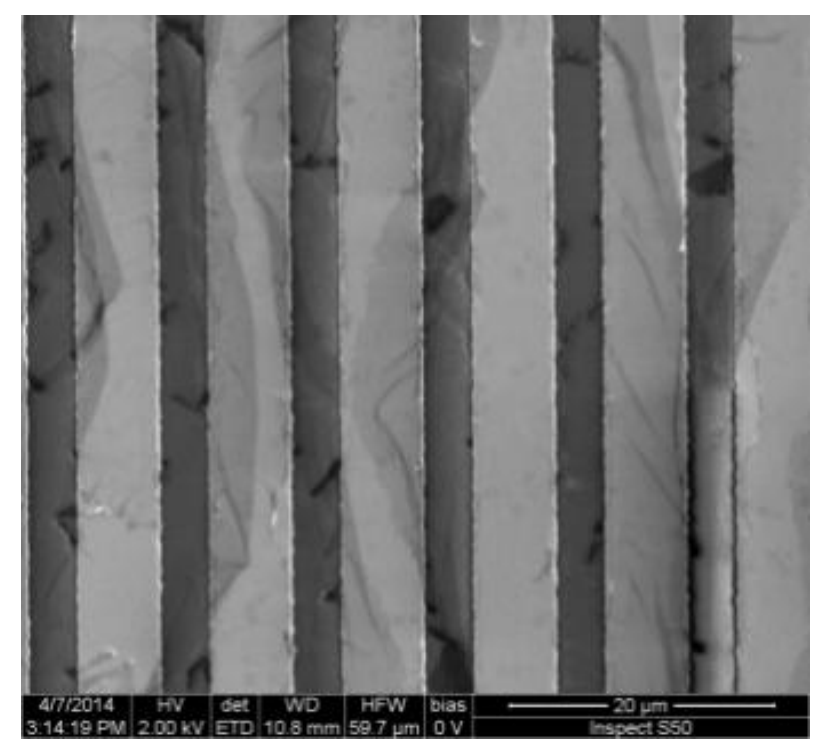

Fig. 5. A fragment of the analyzed graphene structure, obtained in result of applying scaning electron microscopy SEM (type FEI Inspekt S50).

In the investigation, Raman's spectroscopy was also used to charaterize the surface. For this purpose, maps of Raman's spectra were prepared, the areas of which were $10 \mu \mathrm{m} \times 10 \mu \mathrm{m}$. The spectra were gathered with a spectral resolution of $0.05 \mathrm{~cm}^{-1}$. From each spectrum on the map, the half-width (FWHM: Fig. 6a) and the position of the characteristic 2D peak (Fig. 6b) were determined. 

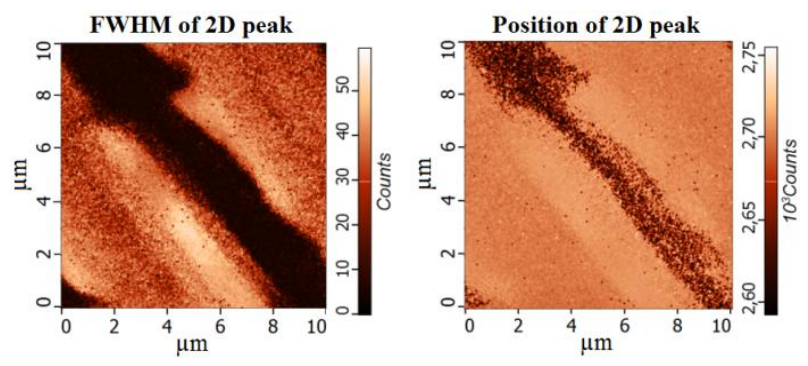

Fig. 6. Half-width (FWHM) and position of the peak 2D.

The comparison between the ratio of intensity of the peaks $2 \mathrm{D}$ to $\mathrm{G}$ and the half-width provides information about the graphene layer. In the area of electrodes, the $2 \mathrm{D} / \mathrm{G}$ ratio is approximately constant (equal to $\sim 1.5$ ). Another situation is observed between the electrodes. In this area, the $2 \mathrm{D} / \mathrm{G}$ ratio is not demined unambiguously. This situation may be due to the suspension of graphene between the electrodes or local damage of the graphene layer.

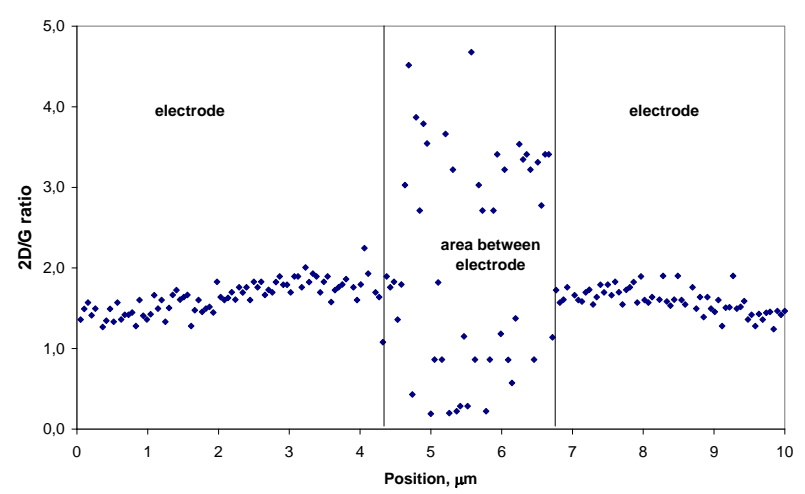

Fig. 7. The ratio of intensity of the peaks $2 \mathrm{D} / \mathrm{G}$ in the function of their position.

The position of the peak 2D permits to assess whether stresses of the compressing or extending type occur in the graphene[12]. Analyzing obtained Raman's spectra, we may find that compressing stresses are acting on the electrodes (shifting the 2D peak towards the higher wave numbers). Between the electrodes, a shift of the wave number may be observed in the direction of lower values, which suggests a graphene layer extension in this place.

In conclusion, the performed investigations have proved that an analysis of a graphene layer between electrodes is not easy. The graphene between electrodes is subject to extending forces. It can be concluded that the graphene is hanging (fully or partially) in the air between the electrodes. Graphene deposited on a surface of gold electrodes is subject to compressing stress.

Actually, investigations concerned the action of external gaseous atmospheres on layers of graphene deposited on different substrates. These investigations ought to provide information about the sensitivity of graphene exposed to low concentrations of selected gases in synthetic air atmosphere.

The work was partially sponsored by the Polish National Science Centre (NCN) within the grant 2012/07/B/ST7/01 471.

\section{References}

[1] T. Pustelny, M. Setkiewicz, S. Drewniak, E. Maciak, A. Stolarczyk, M. Urbańczyk, M. Procek, K. Gut, Z. Oplski, I. Pasternak, W. Strupinski, Bull. Pol. Ac: Tech. 61, 2 (2013).

[2] S.-Y. Hong, J.I. Dadap, N. Petrone, P. Ch. Yeh, J. Hone, R.M. Osgood Jr., Phys. Rev. X 3021014 (2013).

[3] T. Pustelny, M. Setkiewicz, S. Drewniak, E. Maciak, A. Stolarczyk, M. Procek, M. Urbańczyk, K. Gut, Z. Opilski, I. Pasternak, W. Strupinski, Acta Phys. Pol. A 122, 5 (2012).

[4] L. Liao, Y.-Ch. Lin, M. Bao, R. Cheng, J. Bai, Y. Liu, Y. Qu, K.L. Wang. Y. Huang, X. Duan, Nature 467(7313), 305 (2010).

[5] J.-H. Ahn, SPIE Newsroom (2014).

[6] G. Tsoukleri, J. Parthenios, K. Papagelis, R. Jalil, A.C. Ferrari, A.K. Geim, K.S. Novoselov, C. Galiotis, Small 5, 21 (2009).

[7] A. Wysmołek, J. Tworzydło, A. Drabińska, Progress in Physics (in Polish) 62, 3 (2011).

[8] M. Procek, T. Pustelny, A. Stolarczyk, E. Maciak, Bull. Pol. Ac.: Tech. 62, 4 (2014).

[9] T. Ciuk, I. Pasternak, A. Krajewska, J. Sobieski, P. Caban, J. Szmidt, W. Strupinski, Electrochemical Delamination J. Phys. Chem. C 117, (2013).

[10] R.K. Joshi, H. Gomez, F. Alvi, A. Kumar, J. Phys. Chem. C, 114, 14 (2010).

[11] O. Albrektsen, R.L. Eriksen, S.M. Novikov, D. Schall, M. Karl, S.I. Bozhevolnyi, A.C. Simonsen, J. Appl. Phys. 111, 064305 (2012).

[12] K. Grodecki, Electronic Materials (in Polish), 41, 1 (2013).

[13] B. Trauzettel, Progress in Physics (in Polish) 58, 6 (2007).

[14] J. Yu, M. Shafei, K. Ou, K. Shin, W. Wlodarski, A Study of Hydrogen Gas Sensing Performance of Pt/Graphene/GaN Devices (IEEE, 2011).

[15] H. Kim, A.A Abdala, Ch.W. Macosko, Macromolecules 43, 16 (2010).

[16] Y.Y. Wang, Z.H. Ni, T.Yu, Z.X. Shen, H.M. Wang, Y.H. Wu, W. Chen, A.T. Shen Wee, J. Phys. Chem. C 112, 29 (2008).

[17] N. Kim, M.K. Oh, S. Park, S.K. Kim, B.H. Hong, Bull. Korean Chem. Soc. 31, 4 (2010).

[18] K.N. Kudin, B. Ozbas, H.C. Schniepp, R.K. Prud'homme, I.A. Aksay, R. Car, Nano Lett. 8, 1 (2008). 